\title{
Blue Noise Sampling with Controlled Aliasing
}

\author{
DANIEL HECK, THOMAS SCHLÖMER, and OLIVER DEUSSEN \\ University of Konstanz
}

\begin{abstract}
In this article we revisit the problem of blue noise sampling with a strong focus on the spectral properties of the sampling patterns. Starting from the observation that oscillations in the power spectrum of a sampling pattern can cause aliasing artifacts in the resulting images, we synthesize two new types of blue noise patterns: step blue noise with a power spectrum in the form of a step function and single-peak blue noise with a wide zero-region and no oscillations except for a single peak. We study the mathematical relationship of the radial power spectrum to a spatial statistic known as the radial distribution function to determine which power spectra can actually be realized and to construct the corresponding point sets. Finally, we show that both proposed sampling patterns effectively prevent structured aliasing at low sampling rates and perform well at high sampling rates.
\end{abstract}

Categories and Subject Descriptors: I.3.3 [Computer Graphics]: Picture/ Image Generation-Antialiasing; I.4.1 [Image Processing and Computer Vision]: Digitization and Image Capture-Sampling

General Terms: Theory, Experimentation

Additional Key Words and Phrases: Sampling, blue noise, anti-aliasing, power spectrum, radial distribution function, effective Nyquist frequency

\section{INTRODUCTION}

One of the fundamental problems in computer graphics is that high image frequencies cannot always be removed by prefilteringsampling such signals therefore necessarily leads to some form of aliasing. One common solution to reduce the visibility of this aliasing is to employ sampling patterns with a blue noise power spectrum, which prevents conspicuous aliasing artifacts by replacing them with incoherent noise.

Even though the blue noise properties are defined in the frequency domain, most methods for constructing blue noise patterns work in

Authors' addresses: D. Heck (corresponding author), T. Schlömer, and O. Deussen, University of Konstanz, Germany; email: daniel.heck@unikonstanz.de. the spatial domain, usually by placing geometric constraints on the sample positions. The resulting sampling patterns tend to work very well if the image being sampled is dominated by low frequencies. An issue that has been ignored so far, however, is that these sampling patterns can produce artifacts at higher frequencies (Figure 1). Ironically, these artifacts are in fact low-frequency aliasing - exactly the kind of image error blue noise sampling was originally designed to prevent. This kind of aliasing is conceptually similar to moiré patterns, but it also occurs for irregular sampling patterns; we refer to it as structured or coherent aliasing. As suggested by Figure 1, structured aliasing is caused by oscillations in the sampling patterns' power spectra.

These oscillations are not an inherent or necessary feature of blue noise sampling, however, and our goal in this article is to construct sampling patterns with a lower amount of oscillation. As a reference, we define step blue noise as a power spectrum that has the shape of a step function (see the last column of Figure 1). To optimize the blue noise properties of such a spectrum, we show how to derive the maximum frequency at which the step can occur. The point sets corresponding to this maximum frequency differ significantly from the well-known Poisson disk patterns prevalent in computer graphics.

To assess the blue noise qualities of a sampling pattern we introduce two useful measures for the shape of the spectrum: the effective Nyquist frequency $\nu_{\text {eff }}$ and the spectrum oscillation $\Omega$. These two measures suggest two broad categories of blue noise patterns.

- Low aliasing blue noise is characterized by a spectrum with low oscillation, which guarantees that aliasing is mapped to broadband noise. This class contains stochastic and jittered grid sampling as well as the proposed step blue noise. The challenge for this category is to maximize the effective Nyquist frequency without causing oscillations.

- High effective Nyquist blue noise is characterized by a high value of $v_{\text {eff }}$, which guarantees that a large range of low frequencies can be sampled without aliasing. This comes at the cost of more oscillation at higher frequencies and therefore an increased risk of structured aliasing. This class contains most blue noise patterns proposed in recent years. The challenge for this category is to keep the oscillation as low as possible.

For this second category, we design single-peak blue noise sampling patterns whose power spectra are flat except for a single peak. The resulting patterns achieve a good trade-off between $v_{\text {eff }}$ and $\Omega$ : the effective Nyquist frequency is comparable to that of many high-quality blue noise patterns, but by controlling the shape of the spectrum, we can limit the oscillation and hence the amount of structured aliasing that can be introduced during image-plane sampling.

The algorithm used to construct sampling patterns that match a given power spectrum is discussed in Section 4. It is conceptually similar to the recent method by Zhou et al. [2012] in that it exploits the connection between Fourier analysis and spatial statistics to translate the construction problem into the spatial domain. However, in contrast to Zhou et al., our method is based on a one-dimensional statistic called the radial distribution function, which also underlies
Konstanzer Online-Publikations-System (KOPS)

URL: http://nbn-resolving.de/urn:nbn:de:bsz:352-243584 

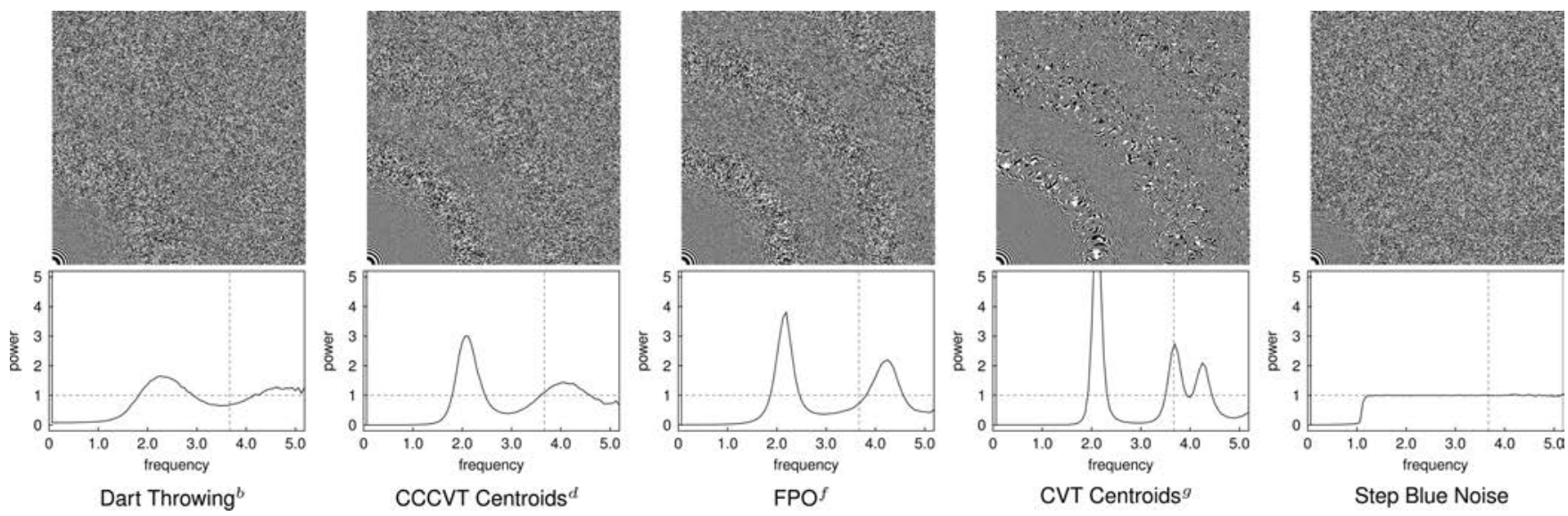

Fig. 1. A bird's eye view of the standard zone plate reveals structured aliasing for blue noise patterns that have peaks in their power spectrum. High-frequency image content is not replaced by white noise (as is the goal of stochastic sampling) but by pseudo-patterns that are conceptually similar to the moiré patterns of regular sampling. Step blue noise patterns, as defined in Section 3.1, consistently prevent such artifacts by cleanly mapping all high frequencies to white noise. (The superscripts refer to the citations in Table 1.)

our theoretical arguments in Section 3. Even though this limits us to radially symmetric spectra, using a one-dimensional statistic makes it easier and more affordable to achieve accurate results.

We evaluate the presented sampling patterns in detail in Section 5 and show that for image-plane sampling, they perform as well or better than many known sampling patters. We think that the ability to directly influence the spectral properties of sampling patterns is a promising area for future research and discuss several interesting open questions in Section 6.

\section{BACKGROUND AND RELATED WORK}

Aliasing is often unavoidable in computer graphics, since many image signals aren't band-limited and exact prefiltering is only rarely possible. Stochastic sampling can reduce the visibility of this aliasing by replacing it with incoherent noise [Dippé and Wold 1985; Cook 1986]. Better results can generally be obtained with blue noise sampling. The term "blue noise" was originally coined by Ulichney [1988] to describe the spectral characteristics of pleasant dithering patterns, but his definition is purely empirical and doesn't directly apply to sampling applications.

Our analysis later in the article builds on Mitchell's work, who was the first to analyze blue noise sampling from a signal processing perspective [Mitchell 1991]. Mitchell attributes the following definition of blue noise to Yellot [1983], who conjectured that aliasing is least conspicuous if:

(1) the power spectrum of the sample points is noisy and without concentrated spikes, and

(2) the spectrum is close to zero for low frequencies.

This is the blue noise definition we will use in the remainder of this article.

\subsection{Constructing Blue Noise Point Sets}

Constructing sampling patterns with blue noise properties is a nontrivial task that has received a lot of attention in the last 20 years [Lagae and Dutré 2008]. The problem is usually approached by arranging points subject to certain geometric constraints such as large mutual distances between samples, centroidal Voronoi tessellations, etc. Constructing blue noise patterns is still an active area of research; a few recent publications are Schmaltz et al. [2010], Fattal [2011], Schlömer et al. [2011], and Chen et al. [2012].

Most construction algorithms work in the spatial domain, even though the blue noise property itself is defined in the Fourier domain. The only construction algorithms we are aware of that take frequency properties into direct account are the paper by Mitsa and Parker [1992] and the recent work by Zhou et al. [2012]. Zhou et al. construct point sets matching a given power spectrum by performing a gradient descent optimization on an energy derived from the autocorrelation function. A similar approach based on simulated annealing was proposed in the context of solid state physics [Rintoul and Torquato 1997]. We present a third variant for constructing point sets with a given spectrum in Section 4.

\subsection{Analysis of Sampling Patterns}

Fourier analysis is the standard method for studying the properties of sampling patterns [Ulichney 1993; Lagae and Dutré 2008]. For a finite set of $n$ points in the unit square, the power spectrum $P(v)$ can be defined as

$$
P(v)=\frac{1}{n}|S(v)|^{2}=\frac{1}{n} \sum_{k, j} e^{-2 \pi i v \cdot\left(x_{k}-x_{j}\right)}
$$

with the sample function $s$ and its Fourier transform $S$

$$
s(x)=\sum_{k=1}^{n} \delta\left(x-x_{k}\right), \quad S(v)=\sum_{k=1}^{n} e^{-2 \pi i v \cdot x_{k}} .
$$

We denote the radial average of the power spectrum by $P(v)$. For conciseness, we use the term "power spectrum" also for this onedimensional profile. For infinite point sets and point processes, the sums must be replaced by appropriate integrals (see Appendix B); in this case, $n$ denotes the number of points per unit area.

Alternatively, point sets can also be characterized using spatial statistics [Illian et al. 2008]. Spatial statistics are widely used in domains that study the arrangement of point-like objects, such as solid state physics, geology, and astronomy, but have seen little use in computer graphics until recently [Wei and Wang 2011; 

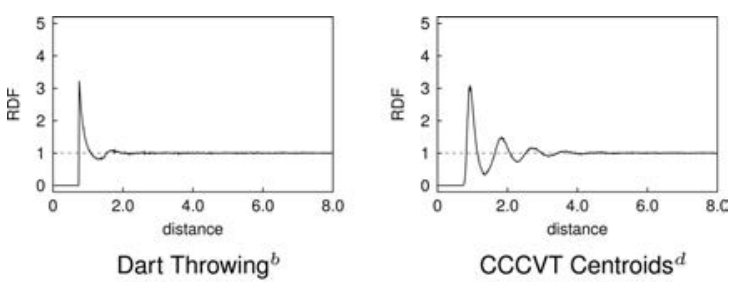

CCCVT Centroids $^{d}$
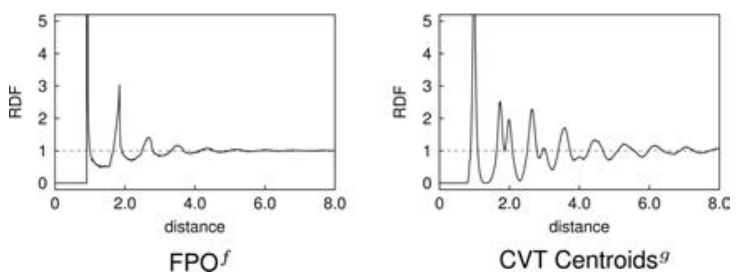

Fig. 2. Radial distribution functions for several of the blue noise patterns in Figure 1.

Zhou et al. 2012]. Two statistics are particularly relevant in our case: the autocorrelation and the Radial Distribution Function (RDF).

The autocorrelation $A_{f}(d)=(f(x) \star f(-x))(d)$ measures the self-similarity of a signal $f$ under translation by a vector $\boldsymbol{d}$. For a sampling pattern $s$, it is easy to show that the power spectrum and the autocorrelation are related by a Fourier transform

$$
P(\boldsymbol{v})=n^{-1} \mathcal{F}\left[A_{s}(\boldsymbol{d})\right] .
$$

Up to normalization, $A_{s}(d)$ is therefore equivalent to the "differential distribution function" proposed by Wei and Wang [2011].

The RDF measures the distribution of point distances and is also known as the pair correlation function. It gives the probability of finding another point at a certain distance from a reference point. Figure 2 shows the RDFs for the power spectra in Figure $1^{1}$. RDF diagrams were used by Lau et al. [2003] to qualitatively illustrate the spatial distribution of points, but we aren't aware of other applications in computer graphics. The RDF $g(r)$ is related to the radial power spectrum $P(v)$ by a Hankel transform

$$
P(v)=1+n \mathcal{H}[g(r)]
$$

(see Appendices A and B). We will use the RDF and Eq. (3) extensively in the remainder of this article.

RDF and autocorrelation both measure the distribution of interpoint distances and are closely related; the main difference is that the $\mathrm{RDF}$ is a function of the scalar distance whereas the autocorrelation is a function of the vector distance. From this perspective, the RDF can be interpreted as the radial average of the function defined in Wei and Wang [2011]. We primarily focus on the RDF in this article because it is easier to handle analytically. Its dependence on absolute distances is not a restriction, since sampling applications generally call for isotropic point sets.

\section{LOW-OSCILLATION BLUE NOISE}

The examples in Figure 1 demonstrate that low-frequency noise occurs in sampled images if the power spectrum oscillates; we will explain this in detail in Section 5. In this section we construct two classes of blue noise patterns that prevent such artifacts by limiting the amount of oscillation.

\footnotetext{
${ }^{1}$ In all figures, we show distances relative to the largest minimum distance of a hexagonal lattice, $d_{\mathrm{hex}}=(2 / \sqrt{3} n)^{1 / 2}$, and frequencies relative to its Nyquist frequency, $v_{\text {hex }}=\sqrt{n} / 2$.
}

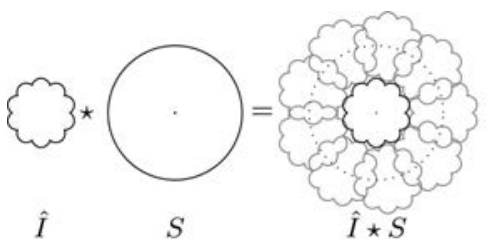

(a)

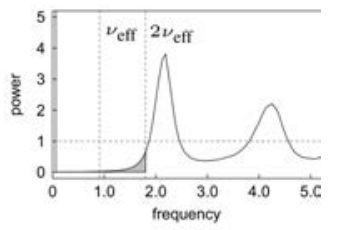

(b)
Fig. 3. (a) The spectrum of the sampled signal $\hat{I}_{s}$ is obtained by convolving the original spectrum with the Fourier transform of the sampling pattern. Aliasing occurs when the replicated spectra $\hat{I}$ overlap the central spectrum on the right-hand side. (b) The effective Nyquist frequency is a measure for the size of the zero region in the power spectrum. It is chosen so that $2 v_{\text {eff }}$ roughly corresponds to the radius of the circle representing $S$ in (a).

\subsection{Step Blue Noise}

We begin with an idealization of blue noise which we call step blue noise and which directly embodies Yellot's blue noise definition mentioned in Section 2: The power spectrum of step blue noise is zero in low frequencies and constant in high frequencies, that is,

$$
P_{\text {step }}\left(v ; v_{0}\right)=n \frac{\delta(v)}{2 \pi v}+H\left(v-v_{0}\right) .
$$

Here we have included the DC peak at the origin and used the Heaviside step function $H$ (refer to Figure 4(a)). Zhou et al. [2012] already constructed a point distribution with a step-like power spectrum, but what is the largest value of $v_{0}$ for which $P_{\text {step }}\left(v ; v_{0}\right)$ is realizable?

There are two necessary conditions a power spectrum $P(v)$ must fulfill to be realizable by a point distribution [Crawford et al. 2003; Uche et al. 2006]: since both $P(v)$ and the associated RDF $g(r)$ are nonnegative by definition, we must have

$$
g(r) \geq 0, \quad P(v) \geq 0 .
$$

Because both functions are linked via a Hankel transform, these two conditions severely limit the range of realizable power spectra. Whether these two conditions are not only necessary but also sufficient is still an open question, but since no counterexamples are known [Torquato and Stillinger 2002] we refer to Eq. (5) as the realizability conditions.

The RDF associated with the step blue noise spectrum (4) is

$$
g_{\text {step }}(r)=1-\frac{2 \pi v_{0}^{2}}{n} \operatorname{jinc}\left(2 v_{0} r\right) .
$$

This follows from Eq. (15) and the Hankel transforms in Appendix A. Solving $g_{\text {step }}(r) \geq 0$ for $v_{0}$, we obtain

$$
v_{0} \leq v_{\max }=\sqrt{n / \pi}
$$

since the jinc function has a maximum of $1 / 2$. This $v_{\max }$ is the highest realizable position of the step: Figure 4(a) demonstrates that for $v_{0}>v_{\max }$, the RDF $g_{\text {step }}$ becomes negative. The only way to move the position of the step further to the right is to increase the sample density $n$.

Figure 4 also shows one resulting point set for $v_{0}=v_{\max }$. Note that the point distribution differs significantly from usual Poisson disk patterns in that it contains many closely spaced point pairs. We will evaluate this sampling pattern in more detail in Section 5.

\subsection{Quantifying Blue Noise}

By comparing the power spectra in Figure 1, we see that step blue noise has a narrower zero region than classic blue noise patterns. 

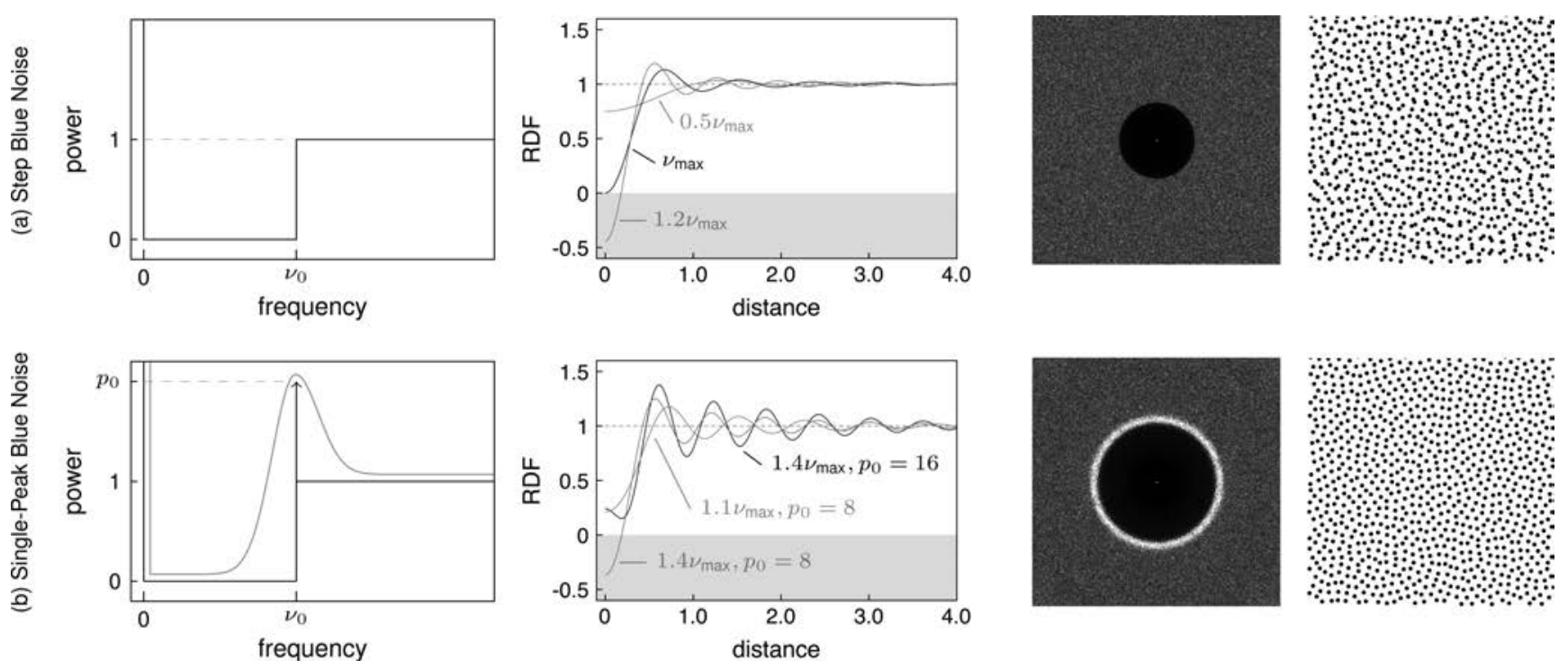

Fig. 4. Radial power spectra, corresponding RDFs, two-dimensional power spectra and cutouts from corresponding points sets for step blue noise and single-peak blue noise. (a) A radial power spectrum that is a perfect step is only possible up to a maximum frequency $v_{\max }$. For frequencies $v_{0}>v_{\max }$ the corresponding RDF becomes negative. (b) To achieve a higher effective Nyquist frequency, we have to allow the power spectrum to go above 1 by introducing a (potentially smoothed) peak around $v_{0}>v_{\max }$. To keep the corresponding RDF positive, we have to increase the height $p_{0}$ of the peak as we increase $v_{0}$.

Increasing the zero region beyond $v_{\max }$ requires us to sacrifice some of the flatness of $P(v)$ in the high-frequency region. We will discuss a controlled way to do this in the next section. As a preparation, we introduce two numerical measures that characterize the shape of the power spectrum: the effective Nyquist frequency $v_{\text {eff }}$ measures the size of the zero region and indicates the range of frequencies that can be represented with almost no aliasing; the oscillation $\Omega$ measures the amount of oscillation in the power spectrum, and therefore the risk of coherent aliasing.

In the theory of uniform sampling, the range of frequencies that can be reconstructed without aliasing is given by the Nyquist frequency $v_{c}=1 / 2 d$, where $d$ is the sample spacing. This direct relationship between sample distances and frequencies that can be reconstructed unfortunately breaks down for nonuniform sampling. As an alternative, we define the equivalent of the Nyquist frequency directly in the frequency domain.

In general, the spectrum of a sampled image $I_{s}$ is given by $\hat{I}_{s}=$ $\hat{I} \star S$, where $\hat{I}$ is the original image spectrum and $S$ the Fourier transform of the sample set as defined in Eq. (2). This relationship is visualized in Figure 3. This diagram suggests that the range of aliasing-free frequencies roughly equals half the radius of the zero region in the power spectrum.

To formalize this idea, we consider the average energy in the power spectrum up to a certain frequency $v$

$$
P_{\mathrm{avg}}(v)=\frac{1}{\pi v^{2}} \int_{\left|v^{\prime}\right|<v} P\left(v^{\prime}\right) \mathrm{d} v^{\prime}
$$

and define the effective Nyquist frequency $\nu_{\text {eff }}$ as the largest frequency so that $P_{\text {avg }}\left(2 v_{\text {eff }}\right)$ stays below a given threshold

$$
v_{\text {eff }}=\max \left\{v: P_{\text {avg }}(2 v) \leq E_{\tau}\right\} .
$$

Ideally, we would like to set $E_{\tau}=0$ to determine which frequencies can be represented without any aliasing, but this is impractical since stochastic sampling patterns always introduce some noise into the low-frequency region. Our reference for choosing $E_{\tau}$ is dart throwing, which has more noise in the low-frequency region than other blue noise patterns. We chose $E_{\tau}=0.1$, which is the lowest threshold for which dart throwing consistently yields a nonzero value for $v_{\text {eff. }}$.

To measure the amount of oscillation of the power spectrum, we use the standard deviation of $P(\boldsymbol{v})$ from the 1-level

$$
\Omega=10\left(\frac{1}{|R|} \int_{R}|P(v)-1|^{2} \mathrm{~d} v\right)^{1 / 2}
$$

Here, $R$ is the integration domain and $|R|$ its area. We exclude the zero region of the power spectrum by integrating over the ring $R=\left\{v: v_{0} \leq|v| \leq v_{1}\right\}$. The inner radius $v_{0}$ is the lowest frequency for which $P\left(\bar{v}_{0}\right)=1$; the outer radius $v_{1}=v_{0}+10 v_{\text {hex }}$ is chosen to cover approximately the first 10 peaks in the power spectrum.

The effective Nyquist frequency is similar to the conventional Nyquist frequency in the sense that frequencies below $v_{\text {eff }}$ can be sampled and reconstructed with little error. Frequencies above $v_{\text {eff }}$, on the other hand, are replaced by aliasing. In this case, the magnitude of $\Omega$ determines whether this noise is, on average, closer to white noise ( $\Omega$ small) or colored noise ( $\Omega$ large).

\subsection{Single-Peak Blue Noise}

The step blue noise patterns from Section 3.1 prevent coherent aliasing by keeping the power spectrum flat, but this comes at a cost: The effective Nyquist frequency of these patterns cannot be higher than $\sqrt{n / 4 \pi}$, which is $56.4 \%$ of the maximum Nyquist frequency $v_{\text {hex }}$ achieved by the hexagonal lattice. In this section, we discuss a class of blue noise patterns that offer a much higher Nyquist frequency (up to $86 \%$ of $v_{\text {hex }}$ ) by introducing a single peak into an otherwise flat power spectrum.

The main problem when constructing blue noise patterns with a higher effective Nyquist frequency is that we cannot choose $P(v)$ arbitrarily due to the constraints imposed by the realizability 

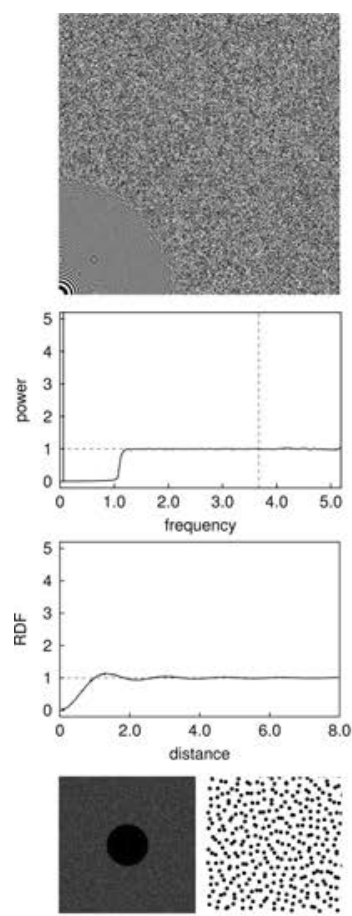

$\nu_{0}=\nu_{\max }, p_{0}=1, \sigma=0$

$\nu_{\text {eff }}=0.58, \Omega=0.01$

(a) Step Blue Noise
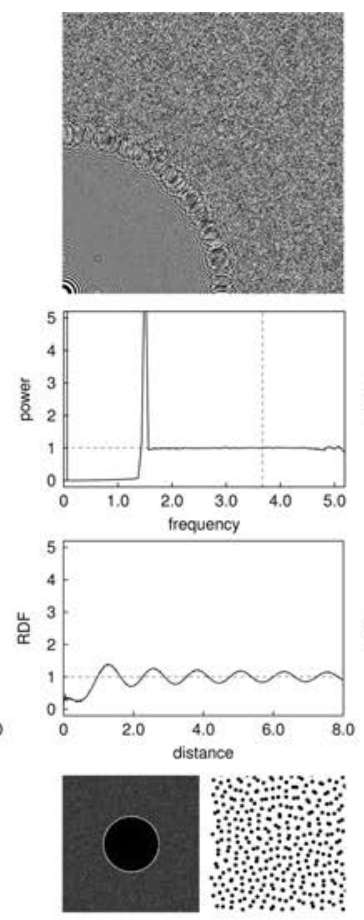

$1.33 \nu_{\max }, p_{0}=16, \sigma=0$ $\nu_{\text {eff }}=0.72, \Omega=1.34$

(b)
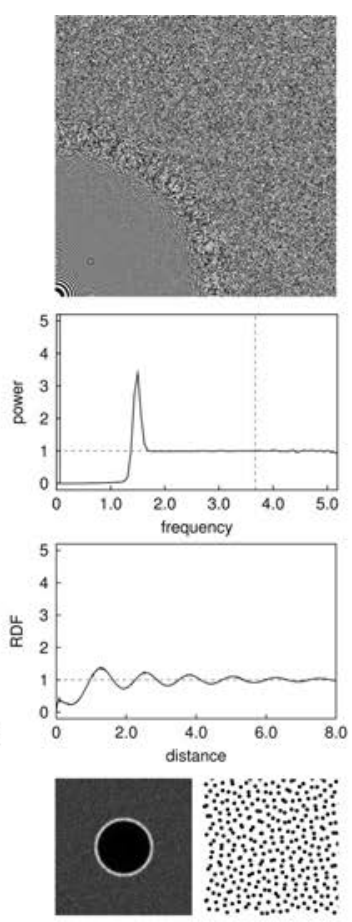

$1.33 \nu_{\max }, p_{0}=16, \sigma=4$ $\nu_{\text {eff }}=0.7, \Omega=1.01$

(c)
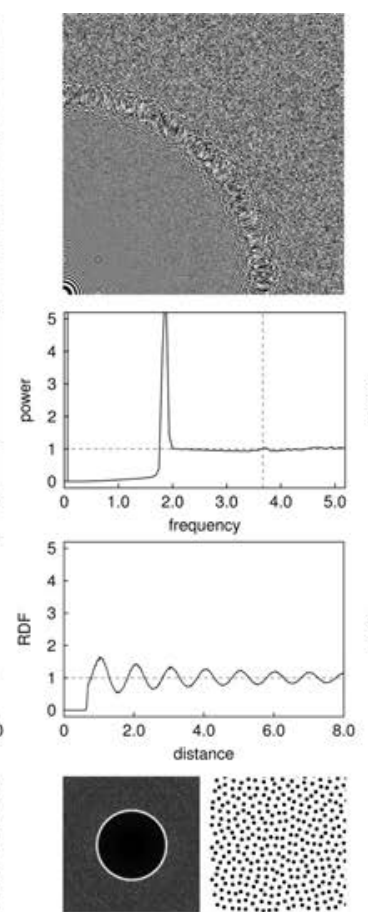

$1.66 \nu_{\max }, p_{0}=20, \sigma=2$ $\nu_{\text {eff }}=0.88, \Omega=1.85$

(d)
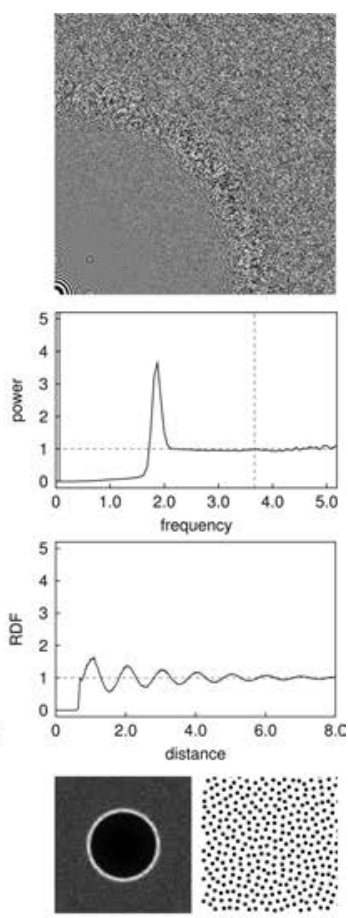

1.66 $\nu_{\max }, p_{0}=20, \sigma=5$ $\nu_{\text {eff }}=0.86, \Omega=1.44$

(e) Single-Peak Blue Noise

Fig. 5. A selection of realizable blue noise patterns with a single peak. (a) Step blue noise with a step of constant power and $v_{\text {eff }}=0.58$. (b) We can increase $v_{\text {eff }}$ by moving energy to higher frequencies - in this case a Dirac peak of height $P\left(v_{0}\right)=16$ - but only at the cost of structured aliasing (visible patterns in the form of rings around $v_{0}$ ). (c) Smoothing the peak to $P\left(v_{0}\right) \approx 4$ yields acceptable aliasing at the cost of a slightly reduced $v_{\text {eff }}$. (d) The highest effective Nyquist frequency we can produce without deviating strongly from a flat spectrum in frequencies below or above $v_{0}$. (e) The best compromise we found between a high $v_{\text {eff }}$ and structured aliasing. The stated parameters are for 4096 points; for other sizes $n$, the parameters $p_{0}$ and $\sigma$ must be scaled by $\sqrt{n / 4096}$.

conditions in Eq. (5). In general, finding power spectra that are realizable is a nontrivial problem because the space of functions that obey the realizability conditions is not easy to parametrize [Giraud and Peschanski 2006; Uche et al. 2006].

We discuss a few approaches to find realizable power spectra in Appendix C, but none of them gives us direct control over the effective Nyquist frequency and the amount of oscillation. As an alternative, we consider the following generalization of the step blue noise spectrum.

$$
P_{\text {peak }}\left(v ; v_{0} ; p_{0}\right)=n \frac{\delta(v)}{2 \pi v}+G_{\sigma} \star\left(p_{0} \delta\left(v-v_{0}\right)+H\left(v-v_{0}\right)\right)
$$

Compared to $P_{\text {step }}$, we add a Dirac peak of power $p_{0}$ at the step frequency $v_{0}$ and convolve this peak and the step function with a Gaussian kernel $G_{\sigma}$ with standard deviation $\sigma$. Figure 4(b) illustrates the shape of this power spectrum. This family of blue noise spectra has three interesting properties.

(1) Aside from the single peak at $v_{0}$, there are no oscillations.

(2) The width and height of the peak can be controlled by adjusting the smoothing radius $\sigma$ and the peak energy $p_{0}$.

(3) The step spectrum $P_{\text {step }}$ is included as a special case.

Not all spectra in this family are realizable, however, and due to the realizability conditions, the parameters cannot be adjusted independently. The main challenge therefore is to find combinations of the three parameters $v_{0}, p_{0}$, and $\sigma$ that are realizable and yield good sampling patterns.

We explored this family of blue noise patterns empirically by searching for configurations for which:

$-v_{0}$ is as high as possible,

- the power spectrum is flat above and below $v_{0}$, that is, $P(v) \approx$ $0, v<v_{0}$ and $P(v) \approx 1, v>v_{0}$, and

- structured aliasing is kept at an acceptable level by appropriate choice of $p_{0}$ and $\sigma$.

Figure 5 shows some of the results we generated. Step blue noise with $v_{0}=v_{\max }$ is shown as a reference in Figure 5(a). We then increase the peak position $v_{0}$ to $1.33 v_{\max }(\mathrm{b})$ and (c) and $1.66 v_{\max }(\mathrm{d})$ and (e). For each of these pairs, we show one result with a narrow peak (small $\sigma$ ) and one with a wide peak (large $\sigma$ ). As in all other experiments in this article, we generated sets of 4096 points in the unit torus and averaged the results over ten such sets.

The more we increase the peak height, the further we are able to push $v_{0}$ and thus the effective Nyquist frequency. If we take this too far, however, strong aliasing can show up in the sampled image. The zone plate renderings at the top of Figure 5 demonstrate that visible patterns emerge once $P\left(v_{0}\right)>4$. We can reduce these artifacts by increasing the amount of smoothing $\sigma$. This has two effects: It decreases the height of the peak and increases its width, which means that aliasing is scattered over a wider range of frequencies. The 
highest effective Nyquist frequency we could produce while keeping $P(v)$ sufficiently flat above and below $v_{0}$ is shown in column (d) of Figure 5.

The best compromise we found between a high effective Nyquist frequency and structured aliasing is shown in Figure 5(e); in the remainder of the article we will refer to it as single-peak blue noise. This configuration yields an effective Nyquist frequency that is comparable to classic blue noise patterns but gets rid of most of the high-frequency oscillation.

\section{SPECTRUM MATCHING ALGORITHM}

Before we evaluate the performance of the step and single-peak blue noise patterns in Section 5, we briefly describe the algorithm we used to construct sampling patterns from given power spectra. As mentioned in Section 2.1, similar algorithms have already been proposed by other researchers. In our tests, the annealing approach by Rintoul and Torquato [1997] converged only slowly and didn't give good results for reasonably large point sets. We compare our approach with Zhou et al. [2012] at the end of this section.

From an algorithmic point of view, our method is very similar to that of Zhou et al.: Both algorithms synthesize the point sets by reformulating the problem in the spatial domain, and both iteratively update the positions of all points by applying a force to each point

$$
\boldsymbol{x}_{i}^{\prime}=x_{i}+h \cdot F_{i}
$$

where $h$ is a step size parameter and $F$ is a force that depends on the current point positions. The main difference between both algorithms is how they calculate the forces in Eq. (7): Zhou et al. propose a force based on gradient descent, whereas ours is motivated geometrically.

Let's describe this approach in more detail. First, the "target" power spectrum to be matched $P_{t}(v)$ is transformed into an equivalent RDF $g_{t}(r)$ using a numerical Hankel transform (Appendix B). Since both functions are one-dimensional, our approach can only synthesize isotropic point sets. The current point set is initialized with a random distribution of points. To evolve the point set towards the target distribution, we let all points attract or repel each other using forces of the form

$$
\boldsymbol{F}_{i}=\sum_{j \neq i} f\left(\left|x_{i}-x_{j}\right|\right) \frac{x_{i}-x_{j}}{\left|x_{i}-x_{j}\right|^{2}} .
$$

The function $f(r)$ determines the degree of attraction or repulsion and is defined as

$$
f(r)=\underbrace{\int_{0}^{r} g(x) \mathrm{d} x}_{G(r)}-\underbrace{\int_{0}^{r} g_{t}(x) \mathrm{d} x}_{G_{t}(r)} .
$$

It may seem surprising that $f$ is defined in terms of the integral of the RDFs. This can be motivated as follows: Since the RDF measures the density of points at a certain distance, $G(r)$ measures the average point density in circular regions of radius $r$. Consider a fixed radius $r_{0}$. If $G\left(r_{0}\right)>G_{t}\left(r_{0}\right)$, the current point distribution contains too many point pairs that are closer than $r_{0}$, compared to the target distribution. In this case the point set as a whole has to spread out, so $f\left(r_{0}\right)$ should be repulsive to make room. Conversely, if $G\left(r_{0}\right)<G_{t}\left(r_{0}\right)$, too many pairs have a distance greater than $r_{0}$, so the points have to move closer together; in this case, $f\left(r_{0}\right)$ should be attractive. In both cases, the choice $f(r)=G(r)-G_{t}(r)$ fulfills this condition.

In each iteration, we first calculate all the forces $\boldsymbol{F}_{i}$ and then update the positions according to Eq. (7). The main parameter during each iteration is the step size $h$, which is chosen adaptively depending on the largest force as in Zhou et al. [2012]. We use an additional temperature parameter $T$ to reduce the step size whenever the optimization gets stuck.

$$
h=T \frac{F_{\max }}{\sqrt{n}}, \quad F_{\max }=\max _{i}\left\|F_{i}\right\|
$$

We use the energy $E=\left\|g(r)-g_{t}(r)\right\|_{2}$ to track the progress of the optimization. The optimization is considered stuck if $E$ hasn't decreased during the last 20 iterations; in this case, the temperature is reduced by a constant factor $\alpha$. The choice of $\alpha$ is a trade-off between accuracy (high value) or faster termination (low value); we used a factor of $\alpha=0.9$ for this article. The algorithm starts with $T=1$ and terminates once the temperature has fallen below $10^{-3}$. Since we use a global force and because the new position of each point only depends on the old position of all other points, moving the points can be easily parallelized.

The number of bins we use for calculating the RDFs and the force function $f$ is set to $n_{\text {bins }}=n$. Significantly fewer bins lower the resolution of the force and can lead to inaccurate results, and significantly more bins lead to noisier RDF estimates. We consider point sets in the unit torus and calculate the RDF for absolute distances in the interval $[0,0.5)$. Using smaller intervals can speed up some of the computations [Wei and Wang 2011], but we have found that this reduces the quality of the results for RDFs that decay slowly.

Since a certain amount of noise is inevitable when estimating RDFs from finite point sets, we optionally smooth the histograms using a Gaussian kernel. There is no simple rule for choosing the optimal width $\sigma$ of the Gaussian, since this involves a trade-off between reducing noise versus keeping relevant information in the RDF. In our experiments, good values for $\sigma$ were between 0 and $16 / n_{\text {bins. }}$. All the results in this article have been generated with $\sigma=8 / n_{\text {bins }}$, and no parameters had to be adjusted manually.

Figure 6 compares the result of our algorithm for the step and the single-peak blue noise profile to the recent approach by Zhou et al. [2012]. The step power spectrum is matched slightly better by our algorithm $(\Omega=0.01)$ than by the method by Zhou et al. ( $\Omega=0.05$ ) but, overall, the results are similar. The single-peak spectrum is more challenging and was matched by our algorithm without many defects in the point distributions while the method by Zhou et al. had problems adapting to the target profile. The average computation time using the CUDA-based implementation by Zhou et al. was $15.7 \mathrm{~s}$ on an NVIDIA Quadro 4000, while our $\mathrm{C}++$ implementation took an average of $69.7 \mathrm{~s}$ on a quad-core $2.8 \mathrm{GHz}$ CPU using all cores.

Judging from the anisotropy plots, it seems that both methods produce slightly anisotropic point sets, most noticeably at low frequencies and at the frequency corresponding to the step in the power spectrum. This behavior can at least partly be attributed to the standard definition of anisotropy [Ulichney 1993; Lagae and Dutré 2008] which measures the relative variance and therefore includes a division by the radial power. The anisotropy is therefore ill-defined if the power spectrum is very close to zero, which is the case for our point sets. We use an alternative statistic to measure the orientational order of a point set in the following section.

\section{EVALUATION}

To evaluate the performance of the low-oscillation sampling patterns constructed in Section 3, we proceed in three stages. First, we compare their main characteristics to other sampling patterns. We then include a theoretical analysis of sampling artifacts which 

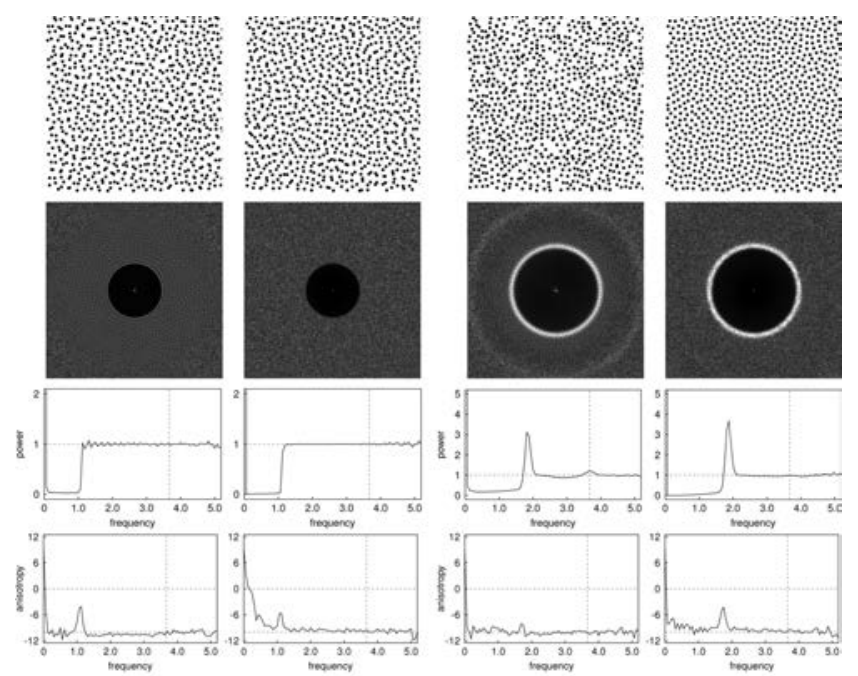

[Zhou et al. 2012] Our Result
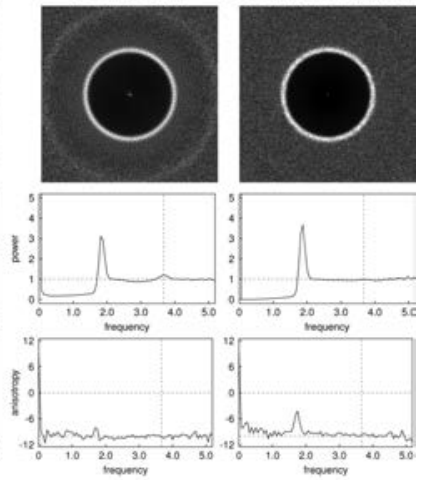

[Zhou et al. 2012]

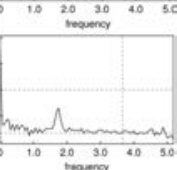

Our Result

Fig. 6. Comparison of matching $P_{\text {step }}$ (left) and $P_{\text {peak }}$ (right) using the method by Zhou et al. [2012] and our approach. Our method matches the target power spectra slightly better and does not show defects in the underlying point distributions. For both methods, the anisotropy shows a noticeable response at the frequency of the step in the corresponding power spectrum.

explains in more detail how the shape of the power spectrum and the frequency of the signal being sampled affect the spectral distribution of aliasing. Finally, we study the performance of step and single-peak blue noise when sampling images both at low and at high sampling rates.

\subsection{Properties}

Table I summarizes several important characteristics of our blue noise sets and compares them with other well-known sampling patterns. We have sorted the sampling patterns into the two categories mentioned in the Introduction: those with low structured aliasing and those with a high effective Nyquist frequency. For reference, we have also included two point sets that are similar to the hexagonal lattice.

The effective Nyquist and oscillation measures from Section 3.2 are shown in the first two columns. We see that for traditional blue noise patterns, a high $v_{\text {eff }}$ comes at the cost of a high oscillation. This interdependence was sometimes referred to as the noise-aliasing trade-off [Dippé and Wold 1985; Glassner 1995]. The point sets constructed in this article demonstrate that it is possible to achieve high values of $v_{\text {eff }}$ with little oscillation, so the trade-off is not strict. Note that practical values for $v_{\text {eff }}$ are slightly larger than theoretical values due to the tolerance introduced by $E_{\tau}$.

The minimal and average nearest-neighbor distance is measured by $\delta_{\min }$ and $\delta_{\text {avg }}$ [Schlömer et al. 2011]. The value of $\delta_{\min }$ corresponds to the Poisson disk radius while $\delta_{\text {avg }}$ roughly measures how uniformly the points are distributed. Our point sets have comparatively low values for both measures. Even though a large separation between samples can be beneficial-as exemplified by the success of Poisson disk patterns - it is not a prerequisite for efficient blue noise sampling.

$Q_{6}$ is the bond orientational order, which measures the similarity of a point distribution to a hexagonal arrangement [Kansal et al. 2000]. Point sets with $Q_{6}<0.5$ have a high amount of "orientational disorder". Both presented point sets are irregular

Table I. Comparison of Several Frequency and Spatial Statistics of Sampling Patterns

\begin{tabular}{|c|c|c|c|c|c|c|c|}
\hline & Method & $v_{\text {eff }}$ & $\Omega$ & $\delta_{\min }$ & $\delta_{\text {avg }}$ & $Q_{6}$ & Note \\
\hline \multirow{4}{*}{ 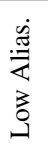 } & Random & 0 & 0.05 & 0.01 & 0.47 & 0.35 & $\mathrm{~F}$ \\
\hline & Jittered Grid ${ }^{a}$ & 0.24 & 0.06 & 0.05 & 0.59 & 0.37 & $\mathrm{~F}$ \\
\hline & Dart Throwing $^{b}$ & 0.58 & 1.52 & 0.76 & 0.80 & 0.42 & \\
\hline & Step Blue Noise & 0.58 & 0.01 & 0.09 & 0.64 & 0.36 & $\mathrm{~F}$ \\
\hline \multirow{5}{*}{ 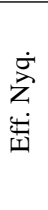 } & Single-Peak Blue Noise & 0.86 & 1.44 & 0.55 & 0.80 & 0.40 & \\
\hline & Kernel Density ${ }^{c}$ & 0.88 & 2.14 & 0.43 & 0.86 & 0.49 & $\mathrm{O}$ \\
\hline & CCCVT Centroids $^{d}$ & 0.89 & 2.34 & 0.75 & 0.88 & 0.53 & $\mathrm{O}$ \\
\hline & El. Halftoning ${ }^{e}$ & 0.89 & 2.49 & 0.74 & 0.88 & 0.52 & $\mathrm{O}$ \\
\hline & $\mathrm{FPO}^{f}$ & 0.90 & 4.64 & 0.93 & 0.93 & 0.47 & $\mathrm{O}$ \\
\hline \multirow{2}{*}{ 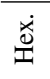 } & CVT Centroids $^{g}$ & 0.98 & 5.35 & 0.80 & 0.94 & 0.85 & $\mathrm{R}, \mathrm{O}$ \\
\hline & Rank-1 ${ }^{h}$ & 1.01 & 12.38 & 0.99 & 0.99 & 0.997 & $\mathrm{R}$ \\
\hline
\end{tabular}

${ }^{a}$ [Cook 1986], ${ }^{b}$ [Lagae and Dutré 2008], ${ }^{c}$ [Fattal 2011], ${ }^{d}$ [Balzer et al. 2009], ${ }^{e}$ [Schmaltz et al. 2010], ${ }^{f}$ [Schlömer et al. 2011], ${ }^{g}$ [Lloyd 1982], ${ }^{h}$ [Dammertz et al. 2008].

The last column marks methods that are largely (F)ree of structured aliasing and methods that are either $(\mathrm{R})$ egular or show strong $(\mathrm{O})$ scillations in their power spectrum.

in this sense. We have found the bond orientational order to be a good alternative to the standard anisotropy measure to assess the regularity of point sets.

\subsection{Sampling: Theoretical Analysis}

In this section we derive an estimate for the error introduced by nonuniform sampling. This analysis will clarify how the shape of the power spectrum affects the amount and the spectral distribution of aliasing in the resulting image and why the low-oscillation patterns constructed in Section 3 perform better than other blue noise patterns. A similar analysis was performed by Dippé and Wold [1985], but they focused on the sampling error for constant functions.

Recall from Section 3.2 that the spectrum of a sampled image $I_{s}$ is given by $\hat{I}_{s}(v)=S \star \hat{I}(v)$, with $S$ being the Fourier transform of the sampling pattern and $\hat{I}$ the Fourier transform of the original image. For sampling patterns with a finite number of points, the function $S$ has two components: a DC peak at the origin and a "noisy" remainder $S^{\prime}$, so we can write

$$
\hat{I}_{s}(v)=\left[n \delta(v)+S^{\prime}(v)\right] \star \hat{I}(v) .
$$

Likewise, we will denote by $P^{\prime}(v)$ the power spectrum without the DC peak. The error introduced by sampling is the difference between the sampled and the original image.

$$
E(v)=\left|\hat{I}_{s}(v) / n-\hat{I}(v)\right|^{2}=\left|S^{\prime} \star \hat{I}(v) / n\right|^{2}
$$

The division by $n$ was introduced to normalize the energy of $I_{s}$. Note that we are primarily interested in the low-frequency region of $E(v)$ up to the Nyquist frequency of the pixel grid, which describes the frequency distribution of aliasing. The high-frequency region of $E(v)$ is removed by the reconstruction process. For a constant image function, Eq. (10) gives us $E(v) \propto\left|S^{\prime}(v)\right|^{2} \propto P^{\prime}(v)$, which is the case studied by Dippé and Wold.

As an important and illustrative class of nonconstant images, we now consider plane cosine waves of the form

$$
I_{f}(x)=\cos (2 \pi f \cdot x), \quad \hat{I}_{f}(v)=\frac{1}{2}(\delta(v-f)+\delta(v+f)) .
$$



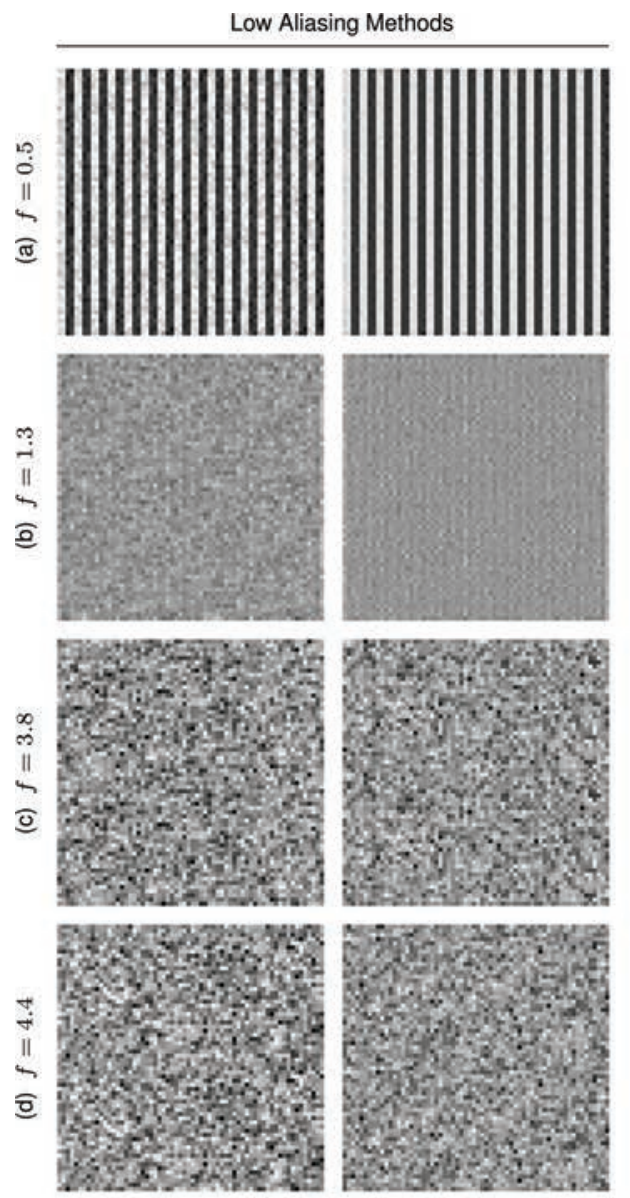

Dart Throwing ${ }^{b}$
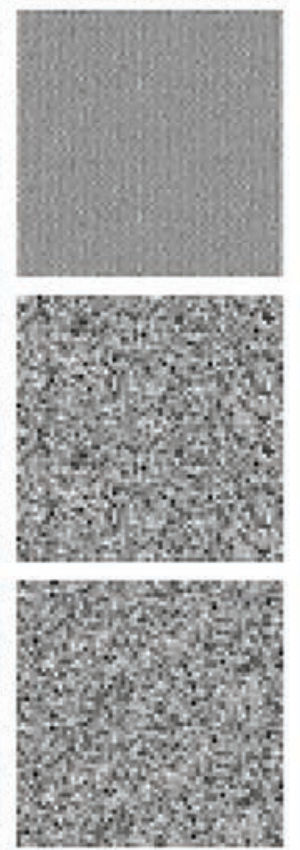

Step Blue Noise
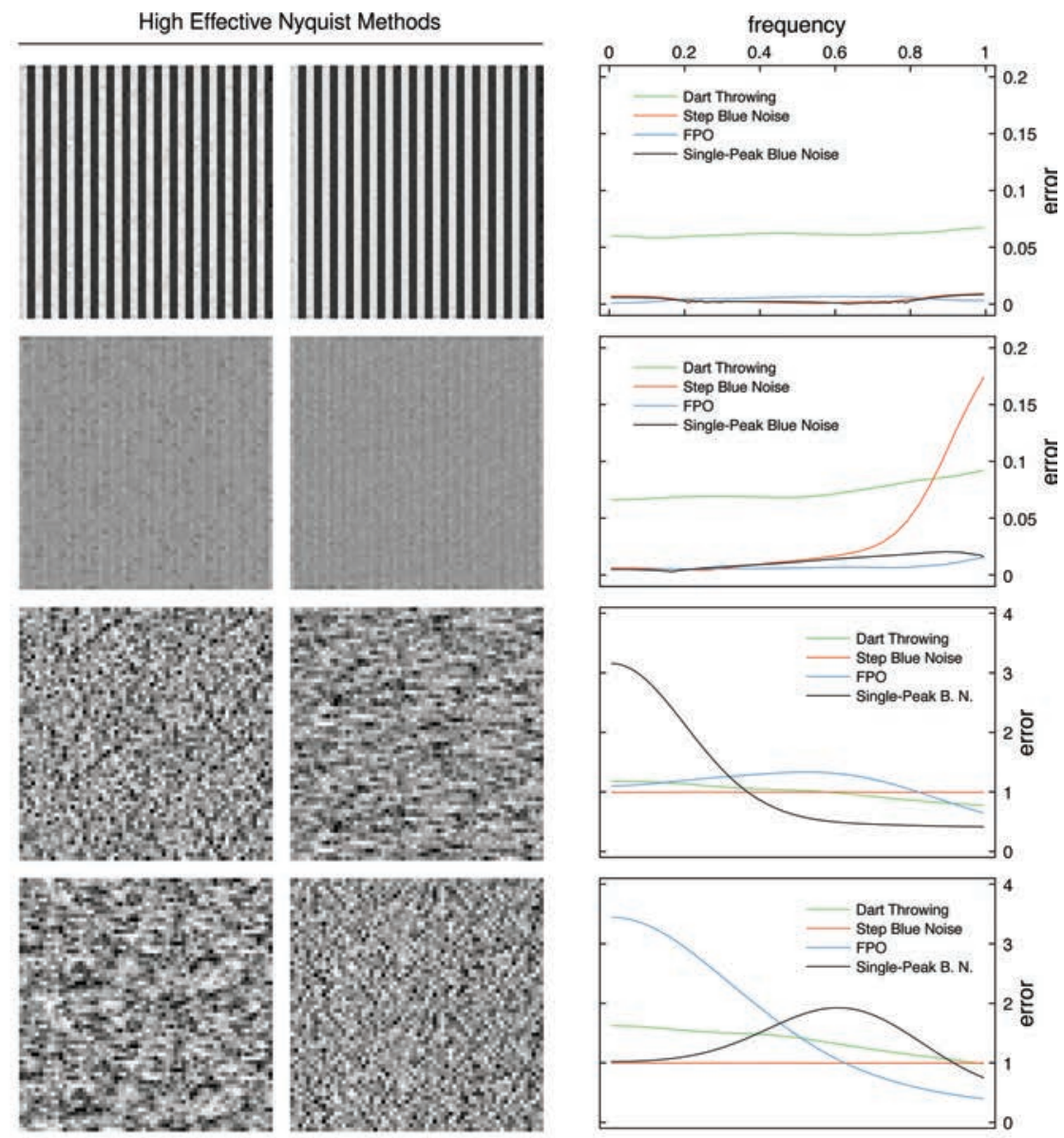

Farthest Point Opt. $f$

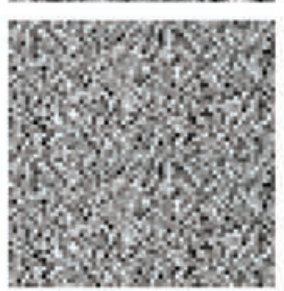

Single-Peak Blue Noise
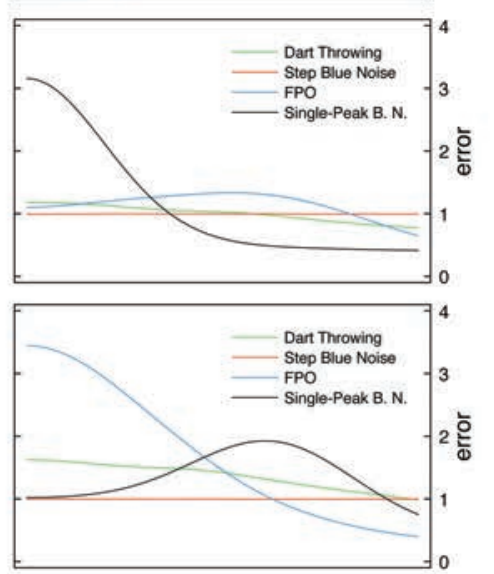

Fig. 7. Illustration of the sampling error for sinusoidals of increasing frequencies. The plots in the rightmost column show the predicted frequency distribution of the noise in the shown images. The frequency axis is relative to $v_{\mathrm{px}}$, the Nyquist frequency of the pixel grid. Note how the shape of the error curve corresponds to noise in the image: flat curves show up as white noise, whereas peaks in the curve lead to different forms of colored noise.

Plugging this into Eq. (10) gives the sampling error

$$
E_{f}(v)=\frac{1}{4 n^{2}}\left|S^{\prime}(v-f)+S^{\prime}(v+f)\right|^{2}
$$

Using the triangle inequality, this error can be bounded from above by the following term which depends only on the power spectrum.

$$
\begin{aligned}
E_{f}(v) \leq & \frac{1}{4 n}\left[P^{\prime}(\boldsymbol{v}-f)+P^{\prime}(\boldsymbol{v}+f)\right. \\
& \left.+2 \sqrt{P^{\prime}(\boldsymbol{v}-f) P^{\prime}(v+f)}\right]
\end{aligned}
$$

Even though this is only an upper bound, we will see that it accurately predicts the frequency characteristics of the sampling error.

A few things can be read off directly from this formula. Since the sampling error is obtained by combining shifted copies of the power spectrum, the result of sampling a cosine with frequency $f$ depends on the shape of $P^{\prime}(v)$ in roughly the following way.

-If $P^{\prime}(v)$ is nonzero, the squared error decreases as $O\left(n^{-1}\right)$ if the number of samples is increased.
-If $P^{\prime}(v)$ is flat around $f$, the error $E_{f}$ is also flat around the origin, that is, the sampling error is rendered as broadband or white noise.

-If $P^{\prime}(v)$ has a peak around frequency $f$, copies of this peak overlap at the origin of $E_{f}$. The width of this peak then determines the appearance of this aliasing: A broad peak also leads to broadband noise, whereas a narrow and high peak produces low-frequency aliasing that appears as structured noise.

This also illustrates why the commonly held opinion that blue noise sampling shifts aliasing to high frequencies is wrong: good blue noise sampling distributes aliasing over all image frequencies.

This is illustrated in Figure 7 which compares the error predicted by Eq. (11) with actual sampled images. If the signal frequency is low, most blue noise patterns perform well since $P^{\prime}(v)$ is close to zero around the origin. Dart throwing is an exception: since its power spectrum is nonzero for all frequencies, $E_{f}(v)$ can never be zero. As we increase the signal frequency, the oscillatory part of $P^{\prime}$ starts to affect $E_{f}$. For step blue noise, the error quickly takes the shape of white noise, as shown by the flat response in the last two rows; the low-amplitude oscillation of dart throwing also leads to broadband noise. 


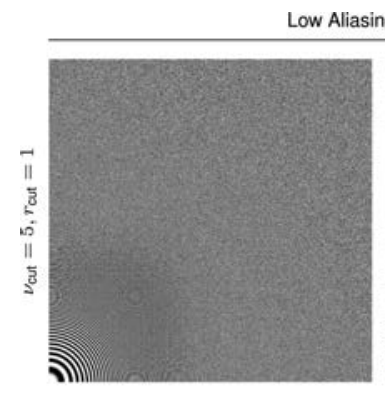

Dart Throwing ${ }^{b}$

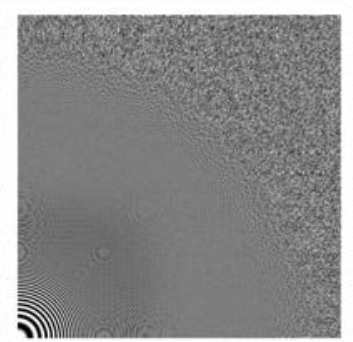

Step Blue Noise

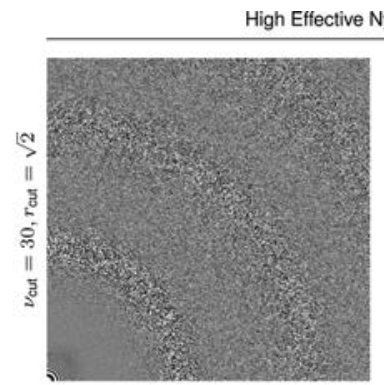

Farthest Point Opt. ${ }^{f}$

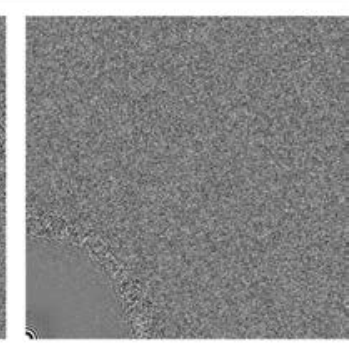

Single-Peak Blue Noise

Fig. 8. A closer look at the behavior of step and single-peak blue noise patterns when used for image-plane sampling. (Left) Like dart throwing, step blue noise produces almost no structured aliasing, but it also keeps low-frequency content much cleaner. (Right) Like traditional blue noise patterns, single-peak blue noise can produce structured aliasing, but only for a narrow range of frequencies around $2 v_{\text {eff }}$. Lower frequencies are represented just as cleanly.

Both single-peak blue noise and Farthest-Point Optimization (FPO) [Schlömer et al. 2011] can cause structured aliasing for certain image frequencies, as demonstrated in the last two rows of Figure 7. Due to the slow decay of $P^{\prime}(v)$ for sampling patterns like FPO, every subsequent peak in the spectrum can potentially cause similar artifacts. Single-peak blue noise, on the other hand, quickly approaches white noise for signal frequencies beyond its peak.

\subsection{Sampling: Practical Evaluation}

5.3.1 Low Sampling Rate. We first evaluate the performance of different sampling patterns when undersampling. As a test image, we use a variant of the common zone plate test function [Mitchell 1990]. For undersampling, the zone plate is preferable to more realistic test images since it shows the response for a wide range of frequencies and aliasing effects are not masked by image features. For all zone plate renderings in this article, we have tiled toroidal sets of 4096 points over the image plane and use a Lanczos filter with a support of width 4 for resampling.

As a replacement for the standard zone plate function $z(r)=$ $\left[1+\cos \left(\alpha r^{2}\right)\right] / 2$, we use the following generalized form that allows us to limit the highest frequency $v_{\text {cut }}$ that can occur and configure the position $r_{\text {cut }}$ of this cutoff frequency in the final image.

$$
z^{\prime}(r)=\frac{1}{2}(1+\cos [v(\alpha r+\phi)])
$$

The frequency $v$, the parameter $\alpha$, and the phase $\phi$ are defined as

$$
\begin{aligned}
v & :=v_{\text {cut }} v_{\mathrm{px}} & & v_{\text {cut }} \geq 0, \\
\alpha & :=\min \left(r / r_{\text {cut }}, 1\right) & & r_{\text {cut }}>0, \\
\phi & :=\max \left(r-r_{\text {cut }}, 0\right) . & &
\end{aligned}
$$

In Figure 1 we already demonstrated that sampling with step blue noise causes almost no structured aliasing for high-frequency signals except for a small amount close to the step. In the left part of Figure 8 we investigate the low-frequency behavior in more detail. Compared to dart throwing, there is significantly less noise in the low-frequency region, which typically contains the most salient image information. Images sampled with stochastic sampling or jittered grid show even more noise in low frequencies.

In the right part of Figure 8 we compare the low-frequency behavior of single-peak blue noise to FPO. We chose FPO to emphasize the effect of heavy oscillations in the power spectrum; the effect is less severe but still visible for other geometrically motivated blue noise methods [Balzer et al. 2009; Schmaltz et al. 2010; Fattal 2011]. Single-peak blue noise shows no structured aliasing
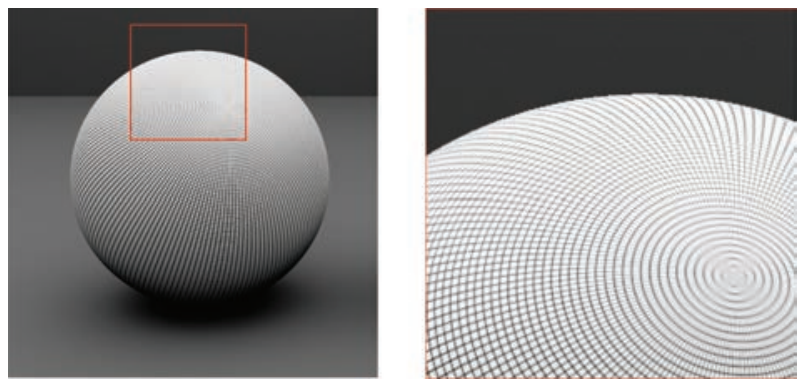

Fig. 9. The scene used to evaluate the performance of sampling patterns at high sampling rates. (Left) Original high-resolution image. (Right) Detail.

beyond the narrow range of frequencies around $2 v_{\text {eff }}$, and even lowfrequency image content appears slightly cleaner.

5.3.2 High Sampling Rate. In most of this article we focused on undersampling, because the risk of undersampling is the primary reason for using stochastic and blue noise sampling instead of regular sampling. It is still important to consider the limiting case, that is, the image quality as we increase the sampling rate. We expect all sampling patterns to improve as the sampling rate increases, but there are measurable differences.

For this evaluation we use a more realistic test image of a three-dimensional scene containing a textured sphere, edges, and smoothly shaded areas [Pharr and Humphreys 2010], and use different sampling patterns to down-sample a high-resolution input to a smaller output (see Figure 9). This is equivalent to image-plane sampling in a raytracer, but easier to control since other error sources (texture filtering, secondary rays) can be ignored. As a quantitative error measure we use the Peak Signal-to-Noise Ratio (PSNR)

$$
\mathrm{PSNR}=10 \cdot \log _{10} \frac{255^{2}}{\mathrm{MSE}}, \quad \mathrm{MSE}=\text { mean square error. }
$$

A PSNR of 50 is comparable to high-quality JPEG compression. At low sampling rates the PSNR would not be an appropriate error measure since it only quantifies the total error, not whether it takes the form of moiré, structured aliasing, or unstructured noise; but in this experiment, we are intentionally oversampling, and the PSNR is only used to quantify the residual noise due to irregular sampling.

The main results are shown in Table II. As expected, the best results were achieved by the regular rank-1 lattice which is close to a perfect hexagonal grid. Of course, the price for this performance 
Table II. PSNR as a Function of the Number of Samples per Pixel (spp)

\begin{tabular}{|c|c|c|c|c|c|}
\hline & Method & $16 s p p$ & $32 s p p$ & 64 spp & $128 s p p$ \\
\hline \multirow{4}{*}{ 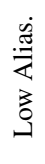 } & Random & 32.2 & 35.4 & 38.5 & 41.7 \\
\hline & Jittered Grid ${ }^{a}$ & 38.2 & 43.5 & 48.9 & 53.9 \\
\hline & Dart Throwing $^{b}$ & 42.4 & 45.9 & 48.9 & 51.6 \\
\hline & Step Blue Noise & 44.4 & 52.2 & 56.6 & 58.3 \\
\hline \multirow{5}{*}{ 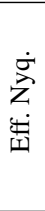 } & Single-Peak Blue Noise & 48.4 & 55.9 & 60.2 & 64.2 \\
\hline & Kernel Density ${ }^{c}$ & 50.3 & 55.9 & 57 & 57.7 \\
\hline & CCCVT Centroids $^{d}$ & 51.9 & 60.8 & 64.8 & 69.9 \\
\hline & El. Halftoning ${ }^{e}$ & 51.6 & 59.6 & 63 & 66.2 \\
\hline & $\mathrm{FPO}^{f}$ & 49.2 & 54.1 & 56.5 & 58.3 \\
\hline \multirow{2}{*}{ 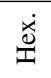 } & CVT Centroids $^{g}$ & 52.6 & 57.2 & 58.3 & 59.3 \\
\hline & Rank-1 ${ }^{h}$ & 55.2 & 64.8 & 66.1 & 75.1 \\
\hline
\end{tabular}

The values are averages over 10 images downsampled to $160 \times 160$ using the respective class of sampling pattern. The reference image is downsampled using a hexagonal grid at $512 \mathrm{spp}$.

is paid as severe moiré artifacts at lower sampling rates. The worst results are obtained by stochastic sampling, which yields visible noise even at 128 samples per pixel.

In the low aliasing category, jittered grid initially performs worse than dart throwing but catches up at high sampling rates. Both are outperformed by step blue noise, however, which produces a significantly higher image quality at all sampling rates. In the high effective Nyquist category, the best results are obtained by CCCVT [Balzer et al. 2009] and electrostatic halftoning [Schmaltz et al. 2010], followed by our single-peak blue noise pattern. Both kernel density [Fattal 2011] and FPO [Schlömer et al. 2011] improve only slowly above $32 \mathrm{spp}$ and eventually perform even worse than step blue noise; we have reproduced this behavior for different test scenes but have been unable to find a good explanation.

\section{CONCLUSION}

In this article, we have revisited the problem of blue noise sampling with a stronger emphasis on the shape of the power spectrum than most recent publications. Starting from the observation that oscillations in the power spectrum can lead to aliasing artifacts, we have proposed two alternatives to standard blue noise patterns:

- step blue noise, which has a step-like power spectrum, and

—single-peak blue noise, which has a single configurable peak but is otherwise flat.

Both blue noise types perform well in image-plane sampling tasks: low frequencies are represented at least as cleanly as with other sampling patterns, but high frequencies are less likely to lead to aliasing since they are mapped to white noise more efficiently.

The main trade-off is in the representation of middle frequencies, and in applications this should determine the choice of sampling pattern. High-oscillation or even regular sampling patterns remain a valid choice if no (structured) aliasing artifacts occur, or if the artifacts that do occur are negligible compared to the increased image quality in medium frequencies. The advantage of sampling patterns like step blue noise is that they guarantee a low amount of coherent aliasing at the cost of a lower effective Nyquist frequency. Patterns like single-peak blue noise trade a better representation of low frequencies for a higher risk of coherent aliasing in middle frequencies. This is basically the classical noise-aliasing trade-off, but the ability to directly shape the power spectrum gives us considerable control over this trade-off.

We are confident that emphasizing the spectral properties of sampling patterns over their geometric properties is a fruitful avenue for further research. Some interesting open questions are as follows.

-We have only studied a very small subset of realizable blue noise spectra, namely step functions and step functions with a single peak at the transition. Differently shaped power spectra might perform even better for image-plane sampling. In particular, it would be interesting to find sampling patterns that can compete with Balzer et al. [2009] at high sampling rates without causing structured aliasing at low sampling rates.

-After writing this article, we have become aware of another recent approach to synthesizing point sets with a given spectrum [Öztireli and Gross 2012], in addition to our algorithm and the one by Zhou et al. [2012]. A thorough comparison of all three methods is still an open question.

-We have not studied the problem of adaptive sampling where the sampling rate is increased locally to oversample critical regions. It should be possible to achieve this by warping the distance metric, similar to the approach by Zhou et al. [2012], but studying the consequences on sampling and reconstruction goes beyond the scope of this article.

\section{APPENDIXES}

\section{A. HANKEL TRANSFORM}

The Fourier transform of a circularly symmetric function $f(r)=$ $f(r, \theta)$ is also circularly symmetric and is called the Hankel transform $\mathcal{H}$ [Bracewell 1999]. This transform is obtained from the standard Fourier transform

$$
F(u, \phi)=\int_{0}^{\infty} r f(r) \int_{0}^{2 \pi} e^{-2 \pi i r u \cos (\theta-\phi)} \mathrm{d} \theta \mathrm{d} r
$$

by rewriting the inner integral using the definition of the Bessel function $J_{0}$

$$
F(u)=\mathcal{H}[f(r)]=2 \pi \int_{0}^{\infty} r f(r) J_{0}(2 \pi r u) \mathrm{d} r .
$$

The inverse Hankel transform is identical to the forward transform,

$$
f(r)=\mathcal{H}[F(u)]=2 \pi \int_{0}^{\infty} u F(u) J_{0}(2 \pi r u) \mathrm{d} u .
$$

The main properties of the Hankel transform follow from the properties of Fourier transforms in two-dimensions, the most important ones we use in the text are the following.

$$
\begin{aligned}
a f(r)+b g(r) & \leftrightarrow a F(u)+b G(u) \\
f(a r) & \leftrightarrow a^{-2} F(u / a) \\
\operatorname{disk}(r) & \leftrightarrow 2 \pi \operatorname{jinc}(2 u) \\
H(r-a) & \leftrightarrow \delta(u) / 2 \pi u-2 \pi a^{2} \operatorname{jinc}(2 u a) \\
\delta(r) / 2 \pi r & \leftrightarrow 1
\end{aligned}
$$

The unit $\operatorname{disk} \operatorname{disk}(r)$ and the Heaviside step function $H(r)$ are defined as

$$
\operatorname{disk}(r)=\left\{\begin{array}{ll}
1 & r \leq 1 \\
0 & r>1
\end{array}, \quad H(r)=\left\{\begin{array}{ll}
0 & r \leq 0 \\
1 & r>0
\end{array} .\right.\right.
$$




\section{B. POWER SPECTRUM AND RDF}

Throughout the article we exploit the close relationship between the RDF and the power spectrum of a point set. To derive this relationship, we modify Eq. (1) by setting $r_{j k}=x_{k}-x_{j}$ and pulling out the $k=j$ terms.

$$
P(\boldsymbol{v})-1=\frac{1}{n} \sum_{k \neq j} e^{-2 \pi i v \cdot r_{j k}}=\int_{\mathbb{R}^{2}} e^{-2 \pi i \boldsymbol{v} \cdot \boldsymbol{r}} \rho(\boldsymbol{r}) \mathrm{d} \boldsymbol{r}
$$

The function $\rho(\boldsymbol{r})$ measures the density of points at distance $\boldsymbol{r}$. To recover Eq. (1), we can set $\rho(\boldsymbol{r})=\sum_{k \neq j} \delta\left(\boldsymbol{r}-\boldsymbol{r}_{j k}\right) / n$, but we usually treat $\rho(\boldsymbol{r})$ as a continuous function. In this form, the power spectrum can be easily generalized to infinite point sets and stationary point processes by appropriate choice of $\rho(r)$.

For isotropic point sets, the density is circularly symmetric, so $\rho(r)=\rho(r)$. In this case, Eq. (13) turns into a Hankel transform.

$$
P(v)-1=\mathcal{H}[\rho(r)]=n \mathcal{H}[g(r)]
$$

The radial distribution function $g(r)=\rho(r) / n$ measures how the distances between pairs of points are distributed. The normalization by $n^{-1}$ ensures that $g(r) \rightarrow 1$ as $r \rightarrow \infty$.

For a Poisson process, points are distributed randomly in the plane with density $n$. In this case the probability of finding another point at distance $r$ from any particle is $n$, so the RDF is constant and the power spectrum is flat with a DC peak at the origin.

$$
g_{\text {Poiss }}(r)=1, \quad P_{\text {Poiss }}(u)=1+n \frac{\delta(u)}{2 \pi u}
$$

For other point distributions, $g(r)$ oscillates around 1 ; this oscillation indicates how much the point set differs from a completely random arrangement. Important information can be read off directly from the RDF, such as the minimum distance between points and the distance at which the points become uncorrelated.

For numerical computations, a different notation is preferable. Since $g(r) \rightarrow 1$ as $r \rightarrow \infty$, the Hankel transform becomes easier to evaluate if we rewrite Eq. (14) as

$$
\begin{aligned}
& P(v)=1+n \mathcal{H}[g(r)-1]+n \frac{\delta(v)}{2 \pi v}, \\
& g(r)=1+n^{-1} \mathcal{H}\left[P(v)-1-n \frac{\delta(v)}{2 \pi v}\right] .
\end{aligned}
$$

Another advantage of this representation is that the main components of a typical power spectrum become evident, namely the DC peak at $v=0$ and the oscillation around 1 , which is due to the Hankel transform of $g(r)-1$.

\section{FINDING REALIZABLE POWER SPECTRA}

We briefly note two ways of constructing realizable power spectra with a higher effective Nyquist frequency that we discarded due to the lack of control over the shape of the spectrum. One option is to subject a step blue noise pattern to an optimization method such as Lloyd's method [Lloyd 1982; Balzer et al. 2009; Chen et al. 2012]. Even though this gradually increases $v_{\text {eff }}$, all of these methods operate in the spatial domain and give us no direct control over the height and shape of oscillations in the power spectrum.

An interesting alternative way to investigate realizable power spectra is to consider the relation between RDF and power spectrum from Eq. (15). If we leave out the DC peak, power spectrum and $\mathrm{RDF}$ become dual in the following sense.

$$
P(v)=1+n \mathcal{H}[g(r)-1], \quad g(r)=1+n^{-1} \mathcal{H}[P(v)-1]
$$
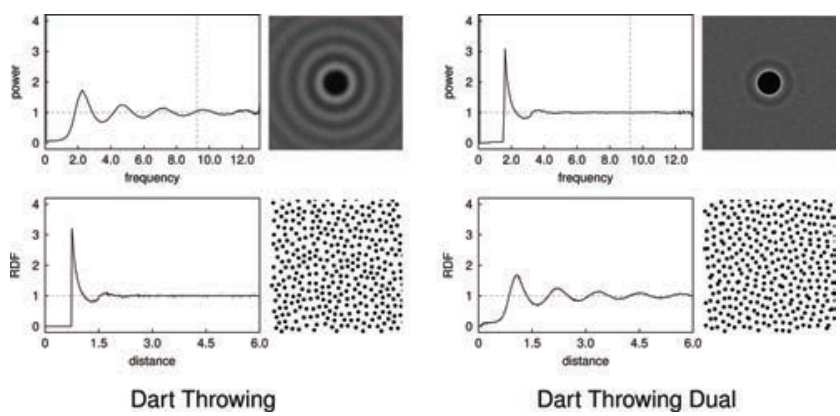

Fig. 10. Comparison of dart throwing to its dual. The two point sets are dual in the sense that the radial power spectrum of the first point set has the shape of the RDF of the second point set and vice versa.

A direct consequence of this duality is that if $g(r)$ is a realizable RDF, then $P(v):=g(v / n)$ is a realizable power spectrum (this follows directly from the scaling properties of the Hankel transform). What is beautiful about this observation is that we immediately have access to large number of realizable RDFs and power spectra, namely those that correspond to classical blue noise patterns (Figures 1 and 2). For example, if we use the RDF of a dart-throwing point set with $g_{\text {dart }}(r)$, we can construct a dual point set such that $P_{\text {dual-dart }}(v)=g_{\text {dart }}(v / n)$ (see Figure 10). Although this approach does not provide a way to control the amount of aliasing induced by the realized point set, it does give the guarantee that the power spectrum is realizable.

\section{ACKNOWLEDGMENTS}

We thank the anonymous reviewers for their detailed feedback on earlier drafts and Victor Ostromoukhov for the discussions on blue noise sampling that ultimately led to this article.

\section{REFERENCES}

Balzer, M., Schlömer, T., AND Deussen, O. 2009. Capacity-constrained point distributions: A variant of Lloyd's method. ACM Trans. Graph. 28, 3, 86:1-8.

Bracewell, R. N. 1999. The Fourier Transform and its Applications 3rd Ed. McGraw-Hill, New York.

CHEN, Z., YuAn, Z., CHOI, Y.-K., LiU, L., AND WANG, W. 2012. Variational blue noise sampling. IEEE Trans. Vis. Comput. Graph. 18, 1784-1796.

CooK, R. L. 1986. Stochastic sampling in computer graphics. ACM SIGGRAPH Comput. Graph. 5, 1, 51-72.

Crawford, J., Torquato, S., and Stillinger, F. H. 2003. Aspects of correlation function realizability. J. Chem. Phys. 119, 14, 7065-7074.

DAmmertZ, H., Keller, A., AND DAMmerTZ, S. 2008. Simulation on rank-1 lattices. In Monte Carlo and Quasi-Monte Carlo Methods, Springer, 205-216.

DipPÉ, M. A. Z. AND WoLD, E. H. 1985. Antialiasing through stochastic sampling. ACM SIGGRAPH Comput. Graph. 19, 3, 69-78.

FATTAL, R. 2011. Blue-noise point sampling using kernel density model. ACM Trans. Graph. 30, 4, 48:1-48:12.

Giraud, B. G. And Peschanski, R. 2006. On positive functions with positive Fourier transforms. Acta Physica Polonica B 37, 331.

GLASSNER, A. S. 1995. Principles of Digital Image Synthesis 1st. Ed. Vol. 1. Morgan Kaufmann, San Francisco, CA.

Illian, J., Penttinen, A., Stoyan, H., And Stoyan, D. 2008. Statistical Analysis and Modelling of Spatial Point Patterns. John Wiley \& Sons. 
Kansal, A. R., Truskett, T. M., AND ToRquato, S. 2000. Nonequilibrium hard-disk packings with controlled orientational order. J. Chem. Phys. 113, 12, 4844.

LAGAE, A. AND DutRÉ, P. 2008. A comparison of methods for generating Poisson disk distributions. Comput. Graph. Forum 27, 1, 114-129.

LaU, D. L., Ulichney, R., AND ARCE, G. R. 2003. Blue- and green-noise halftoning models. IEEE Signal Process. Mag. 20, 4, 28-38.

LLOYD, S. P. 1982. Least square quantization in PCM. IEEE Trans. Inf. Theory 28, 2, 129-137.

Mitchell, D. P. 1990. The anti-aliasing problem in ray tracing. Advanced topics in ray tracing. In ACM SIGGRAPH Course Notes.

Mitchell, D. P. 1991. Spectrally optimal sampling for distribution ray tracing. ACM SIGGRAPH Comput. Graph. 91, 25, 4, 157-164.

Mitsa, T. AND PARKER, K. J. 1992. Digital halftoning technique using a blue-noise mask. J. Opt. Soc. Amer. A 9, 11, 1920-1929.

OztiReli, A. C. AND Gross, M. 2012. Analysis and synthesis of point distributions based on pair correlation. ACM Trans. Graph. 31, 6, 170:1-170:10.

Pharr, M. and Humphreys, G. 2010. Physically Based Rendering: From Theory to Implementation 2nd Ed. Morgan Kaufmann Publishers.

Rintoul, M. D. AND TORQuATO, S. 1997. Reconstruction of the structure of dispersions. J. Colloid Interface Sci. 186, 467-476.
Schlömer, T., Heck, D., ANd Deussen, O. 2011. Farthest-point optimized point sets with maximized minimum distance. In Proceedings of the ACM SIGGRAPH Symposium on High Performance Graphics. 135154.

Schmaltz, C., Gwosdek, P., Bruhn, A., AND Weickert, J. 2010. Electrostatic halftoning. Comput. Graph. Forum 29, 8, 2313-2327.

Torquato, S. AND Stillinger, F. H. 2002. Controlling the short-range order and packing densities of many-particle systems. J. Phys. Chem. B $106,33,8354-8359$.

UChe, O. U., STILLINGer, F. H., AND ToRquato, S. 2006. On the realizability of pair correlation functions. Physica A 360, 21, 21-36.

Ulichney, R. A. 1988. Dithering with blue noise. Proc. IEEE 76, 1, 5679.

Ulichney, R. A. 1993. Digital Halftoning. MIT Press.

WEI, L.-Y. AND WANG, R. 2011. Differential domain analysis for nonuniform sampling. ACM Trans. Graph. 30, 4, 50:1-50:10.

YelLOT, JR., J. I. 1983. Spectral consequences of photoreceptor sampling in the rhesus retina. Science 221, 382-385.

Zhou, Y., Huang, H., Wei, L.-Y., AND Wang, R. 2012. Point sampling with general noise spectrum. ACM Trans. Graph. 31, 4, 76:1-76:11. 